\title{
Set-Valued Non-Linear Random Implicit Quasivariational Inclusions
}

\author{
Salahuddin, Mohammad Kalimuddin Ahmad \\ Department of Mathematics, Aligarh Muslim University, Aligarh, India \\ Email: salahuddin12@mailcity.com, ahmad_kalimuddin@yahoo.co.in
}

Received April 18, 2012; revised May 24, 2012; accepted June 2, 2012

\begin{abstract}
In this paper, we propose iterative algorithms for set valued nonlinear random implicit quasivariational inclusions. We define the related random implicit proximal operator equations and establish an equivalence between them. Finally, we prove the existence and convergence of random iterative sequences generated by random iterative algorithms.
\end{abstract}

Keywords: Set Valued Nonlinear Random Implicit Quasivariational Inclusions; Random Implicit Proximal Operator Equations; Equivalence Relations; Random Iterative Algorithms; Measurable Spaces; Separable Real Hilbert Spaces; Random Strongly Monotone; Random Relaxed Monotone; Random Lipschitz Continuous Mappings

\section{Introduction}

The theory of variational inequality provides a natural and elegant framework for study of many seemingly unrelated free boundary value problems arising in various branches of engineering and mathematical sciences. Variational inequalities have many deep results dealing with nonlinear partial differential equations which play important and fundamental role in general equilibrium theory, economics, management sciences and operations research, see [1-3].

The quasi variational inequalities have been introduced by Bensoussan and Lions [1] and closely related to contact problems with friction in electrostatics and nonlinear random equations frequently arise in biological, physical and system sciences [4,5]. With the emergence of probabilistics functional analysis, the study of random operators became a central topic of this discipline $[4,5]$. The theory of resolvent operators introduced by Brezis [2] is closely related to the variational inequality problems; for applications we refer to [6-8].

Motivated by recent research work on random variational inequalities [9-14], in this paper we consider a class of set valued nonlinear random implicit quasi variational inclusions and a class of random proximal operator equations and establish an equivalence between them. We use the equivalence to suggest and analyzed some iterative algorithms for finding approximate solution of (1). Further we prove the existence of solution of this class of problem and discuss the convergence of iterative sequences generated by these random iterative algorithms.

\section{Preliminaries}

Let $(\Omega, \Sigma)$ be a measurable space and $H$ a separable real Hilbert space with inner product $\langle. .$.$\rangle and norm \|$.$\| .$ We denote $\mathcal{B}(H), 2^{H}$ and $C(H)$ the class of Borel $\sigma$ field in $H$, family of all nonempty power subsets of $H$ and the family of all nonempty compact subsets of $H$ respectively.

Definition 1. A mapping $x: \Omega \rightarrow H$ is called measurable if for any $B \in \mathcal{B}(H),\{t \in \Omega, x(t) \in B\} \in \Sigma$.

Definition 2. A mapping $T: \Omega \times H \rightarrow H$ is called a random operator if for any $x \in H, T(t, x)=x(t)$ is measurable.

Definition 3. A random operator $T$ is said to be continuous if for any $t \in \Omega$, the mapping $T(t, \cdot): H \rightarrow H$ is continuous.

Given random set valued mappings

$T, V, G, P, E: \Omega \times H \rightarrow 2^{H}$ and $f, g, m: \Omega \times H \rightarrow H$ are the single valued mappings. Let $A: \Omega \times H \times H \rightarrow 2^{H}$ be a random set valued mapping such that for each fix $t \in \Omega, v(t) \in H, A(., v(t), t): H \times \Omega \rightarrow 2^{H}$ is a maximal monotone mapping with

$$
\begin{aligned}
& g(t, x(t))-m(t, y(t)) \in \operatorname{dom}(A(., v(t), t)), \\
& \text { for all } t \in \Omega, x, y \in H .
\end{aligned}
$$

Throughout this paper, we will consider the following set valued nonlinear random implicit quasivariational inclusions for finding $t \in \Omega, x(t) \in H$,

$$
\begin{array}{ll}
v(t) \in T(t, x(t)), \quad a(t) \in V(t, x(t)), & b(t) \in G(t, x(t)), \\
w(t) \in P(t, x(t)), \quad u(t) \in E(t, x(t)) & \text { such that }
\end{array}
$$




$$
\begin{aligned}
& 0 \in A(g(t, x(t))-m(t, u(t)), v(t), t) \\
& +f(t, w(t))-N(a(t), b(t), t),
\end{aligned}
$$

where $N: H \times H \times \Omega \rightarrow H$ be the bifunction.

In deterministic case, the problem (1) is equivalent to the problem of the Ding and Park [15].

For a suitable choice of the operators $A, g, m, f, N, T, V$, $G, P$ and $E$ a number of known classes of variational inequalities, quasivariational inclusions can be obtained as special cases of problem, studied previously by many authors including Hassouni and Moudafi [16], Huang [17], Uko [18], Verma [13], Salahuddin [19], and Salahuddin and Ahmad [20].

Definition 4. If $A$ is maximal monotone operator on $H$, then for a given constant $\eta>0$ the proximal operator associated with $A$ is defined by

$$
J_{A}(x)=(I+\eta A)^{-1}(x) \text {, for all } x \in H,
$$

where $I$ is the identity operator. It is also known that the operator $A$ is maximal monotone if and only if the proximal operator $J_{A}$ is defined everywhere on the space. Furthermore, the proximal operator $J_{A}$ is single-valued and nonexpansive, i.e. for all $x, y \in H$,

$$
\left\|J_{A}(x)-J_{A}(y)\right\| \leq\|x-y\| .
$$

Remark 1. Since the operator $A(. .$.$) is a maximal$ monotone operator with respect to the first argument, we define

$$
J_{A(x)}(x)=(I+\eta A(x))^{-1}(x) \text {, for all } x \in H,
$$

the generalized proximal operator associated with $A(., x)=A(x)$.

Related to the problem (1), we consider the problem of finding $t \in \Omega, \quad x(t), z(t) \in H, \quad v(t) \in T(t, x(t))$,

$a(t) \in V(t, x(t)), \quad b(t) \in G(t, x(t)), \quad w(t) \in P(t, x(t))$, $u(t) \in E(t, x(t))$ such that

$$
f(t, w(t))+\eta^{-1}(t) R_{A(v(t), t)}(z(t))=N(a(t), b(t), t)
$$

where $\eta: \Omega \rightarrow(0, \infty)$ be the measurable function and $R_{A(v(t), t)}=I-J_{A(v(t), t)}$.

Here $I$ stand for an identity operator and

$J_{A(v(t), t)}=(I+\eta(t) A(v(t), t))^{-1}$ is the random proximal operator. Equation of the type (2) is called random implicit proximal operator equations.

\section{Random Iterative Algorithm}

In this section, we prove results which will establish eqivalence between the problems (1) and (2). Then we construct a number of iterative algorithms for solving problem (1).

Lemma 1. If $t \in \Omega, x(t) \in H, v(t) \in T(t, x(t))$, $a(t) \in V(t, x(t)), \quad b(t) \in G(t, x(t)), \quad w(t) \in P(t, x(t))$, $u(t) \in E(t, x(t))$ is a random solution set of problem (1) if and only if $t \in \Omega, x(t) \in H, v(t) \in T(t, x(t))$, $a(t) \in V(t, x(t)), \quad b(t) \in G(t, x(t)), \quad w(t) \in P(t, x(t))$, $u(t) \in E(t, x(t))$ such that

$$
\begin{aligned}
& g(t, x(t))= \tau(t) m(t, u(t)) \\
&+\tau(t) J_{A(v(t), t)} {[g(t, x(t))-m(t, u(t))-\eta(t)\{f(t, w(t))} \\
&-N(a(t), b(t), t)\}] \\
&+(1-\tau(t)) g(t, x(t))
\end{aligned}
$$

where $\eta: \Omega \rightarrow(0, \infty)$ and $\tau: \Omega \rightarrow(0, \infty)$ are two measurable functions and $J_{A(v(t), t)}=(I+\eta(t) A(v(t), t))^{-1}$, here $I$ stand for identity functions.

Proof. Let $t \in \Omega, x(t) \in H, v(t) \in T(t, x(t))$, $a(t) \in V(t, x(t)), \quad b(t) \in G(t, x(t)), \quad w(t) \in P(t, x(t))$, $u(t) \in E(t, x(t))$ be the random solution set of (1). Then for a given measurable function $\eta: \Omega \rightarrow(0, \infty)$,

$$
\begin{aligned}
(1) & \Leftrightarrow 0 \in \eta(t)(f(t, w(t))-N(a(t), b(t), t))+\eta(t) A(g(t, x(t))-m(t, u(t)), v(t), t) \\
& \Leftrightarrow 0 \in-(g(t, x(t))-m(t, u(t))-\eta(t)(f(t, w(t))-N(a(t), b(t), t))) \\
& +(I+\eta(t) A(v(t), t))(g(t, x(t))-m(t, u(t))) \\
& \Leftrightarrow g(t, x(t))-m(t, u(t))=(I+\eta(t) A(v(t), t))^{-1}[g(t, x(t))-m(t, u(t))-\eta(t)(f(t, w(t))-N(a(t), b(t), t))] \\
& \equiv J_{A(v(t), t)}[g(t, x(t))-m(t, u(t))-\eta(t)\{f(t, w(t))-N(a(t), b(t), t)\}] \\
& \Leftrightarrow \tau(t)(g(t, x(t))-m(t, u(t)))=\tau(t) J_{A(v(t), t)}[g(t, x(t))-m(t, u(t))-\eta(t)\{f(t, w(t))-N(a(t), b(t), t)\}] \\
& \Leftrightarrow g(t, x(t))=\tau(t) m(t, u(t)) \\
& +\tau(t) J_{A(v(t), t)}[g(t, x(t))-m(t, u(t))-\eta(t)\{f(t, w(t))-N(a(t), b(t), t)\}]+(1-\tau(t)) g(t, x(t)),
\end{aligned}
$$


where $\tau: \Omega \rightarrow(0, \infty)$ be the measurable mapping. This completes the proof.

Theorem 1. The random problem (1) has a random solution set $t \in \Omega, \quad x(t), z(t) \in H, \quad v(t) \in T(t, x(t))$, $a(t) \in V(t, x(t)), \quad b(t) \in G(t, x(t)), w(t) \in P(t, x(t))$, $u(t) \in E(t, x(t))$ if and only if random problem (2) has a random solution set $t \in \Omega, x(t), z(t) \in H$, $v(t) \in T(t, x(t)), a(t) \in V(t, x(t)), b(t) \in G(t, x(t))$, $w(t) \in P(t, x(t)), u(t) \in E(t, x(t))$ where

$$
g(t, x(t))=m(t, u(t))+J_{A(v(t), t)}(z(t))
$$

and

$$
\begin{aligned}
& z(t)=g(t, x(t)) \\
& -m(t, u(t))-\eta(t)(f(t, w(t))-N(a(t), b(t), t)) .
\end{aligned}
$$

Proof. Let $t \in \Omega, x(t), z(t) \in H, v(t) \in T(t, x(t))$, $a(t) \in V(t, x(t)), \quad b(t) \in G(t, x(t)), \quad w(t) \in P(t, x(t))$, $u(t) \in E(t, x(t))$ be a random solution set of (1). Then for a measurable function $\eta: \Omega \rightarrow(0, \infty)$,

$$
\begin{aligned}
& (1) \Leftrightarrow 0 \in \eta(t) A(g(t, x(t))-m(t, u(t)), v(t), t) \\
& +\eta(t) f(t, w(t))-\eta(t) N(a(t), b(t), t) \\
& \Leftrightarrow 0 \in g(t, x(t))-m(t, u(t)) \\
& +\eta(t) A(g(t, x(t))-m(t, u(t)), v(t), t) \\
& -[g(t, x(t))-m(t, u(t)) \\
& -\eta(t)(f(t, w(t))-N(a(t), b(t), t))] \\
& \Leftrightarrow 0 \in(I+\eta(t) A(v(t), t))(g(t, x(t))-m(t, u(t))) \\
& -[g(t, x(t))-m(t, u(t)) \\
& -\eta(t)(f(t, w(t))-N(a(t), b(t), t))] \\
& g(t, x(t))-m(t, u(t)) \\
& =(I+\eta(t) A(v(t), t))^{-1} \\
& \cdot[g(t, x(t))-m(t, u(t)) \\
& -\eta(t)(f(t, w(t))-N(a(t), b(t), t))] \\
& \equiv J_{A(v(t), t)}[g(t, x(t))-m(t, u(t)) \\
& -\eta(t)(f(t, w(t))-N(a(t), b(t), t))] .
\end{aligned}
$$

Take,

$$
\begin{aligned}
z(t) & =g(t, x(t)) \\
& -m(t, u(t))-\eta(t)(f(t, w(t))-N(a(t), b(t), t)) .
\end{aligned}
$$

Then,

$$
g(t, x(t))-m(t, u(t))=J_{A(v(t), t)}(z(t)) .
$$

Thus,

$$
\begin{aligned}
& z(t)=J_{A(v(t), t)}(z(t)) \\
& -\eta(t)(f(t, w(t))-N(a(t), b(t), t)), \\
& \eta^{-1}(t)\left(I-J_{A(v(t), t)}\right)(z(t)) \\
& =-f(t, w(t))+N(a(t), b(t), t) .
\end{aligned}
$$

That is,

$$
f(t, w(t))+\eta^{-1}(t) R_{A(v(t), t)}(z(t))=N(a(t), b(t), t)
$$

where $R_{A(v(t), t)}=I-J_{A(v(t), t)}$, completing the proof of Theorem 1.

Theorem 1 implies that random problems (1) and (2) are equivalent, which allows us to suggest a number of iterative algorithms for solving problem (1). For a suitable rearrangement of the terms of the random Equation (2), we suggest the following algorithms:

1) The random problem (2) can be written as

$$
R_{A(v(t), t)}(z(t))=-\eta(t)(f(t, w(t))-N(a(t), b(t), t))
$$

which implies that

$$
\begin{aligned}
z(t) & =J_{A(v(t), t)}(z(t)) \\
& -\eta(t)(f(t, w(t))-N(a(t), b(t), t)) .
\end{aligned}
$$

This random fixed point formulation allow us to suggest the following random iterative algorithm.

Algorithm 1. For given $t \in \Omega, x_{0}(t), z_{0}(t) \in H$, $v_{0}(t) \in T\left(t, x_{0}(t)\right), \quad a_{0}(t) \in V\left(t, x_{0}(t)\right)$, $b_{0}(t) \in G\left(t, x_{0}(t)\right), \quad w_{0}(t) \in P\left(t, x_{0}(t)\right)$, $u_{0}(t) \in E\left(t, x_{0}(t)\right)$, compute $\left\{z_{n}(t)\right\},\left\{x_{n}(t)\right\}$, $\left\{v_{n}(t)\right\},\left\{a_{n}(t)\right\},\left\{b_{n}(t)\right\},\left\{w_{n}(t)\right\}$ and $\left\{u_{n}(t)\right\}$ by random iterative schemes,

$$
\begin{aligned}
& \quad g\left(t, x_{n}(t)\right)=m\left(t, u_{n}(t)\right)+J_{A\left(v_{n}(t), t\right)}\left(z_{n}(t)\right), \\
& v_{n}(t) \in T\left(t, x_{n}(t)\right), \\
& \left\|v_{n+1}(t)-v_{n}(t)\right\| \leq \mathcal{H}\left(T\left(t, x_{n+1}(t)\right), T\left(t, x_{n}(t)\right)\right), \\
& a_{n}(t) \in V\left(t, x_{n}(t)\right) \\
& \left\|a_{n+1}(t)-a_{n}(t)\right\| \leq \mathcal{H}\left(V\left(t, x_{n+1}(t)\right), V\left(t, x_{n}(t)\right)\right), \\
& b_{n}(t) \in G\left(t, x_{n}(t)\right) \\
& \left\|b_{n+1}(t)-b_{n}(t)\right\| \leq \mathcal{H}\left(G\left(t, x_{n+1}(t)\right), G\left(t, x_{n}(t)\right)\right), \\
& w_{n}(t) \in P\left(t, x_{n}(t)\right), \\
& \left\|w_{n+1}(t)-w_{n}(t)\right\| \leq \mathcal{H}\left(P\left(t, x_{n+1}(t)\right), P\left(t, x_{n}(t)\right)\right), \\
& u_{n}(t) \in E\left(t, x_{n}(t)\right), \\
& \left\|u_{n+1}(t)-u_{n}(t)\right\| \leq \mathcal{H}\left(E\left(t, x_{n+1}(t)\right), E\left(t, x_{n}(t)\right)\right),
\end{aligned}
$$




$$
\begin{aligned}
z_{n+1}(t) & =g\left(t, x_{n}(t)\right)-m\left(t, u_{n}(t)\right) \\
& -\eta(t)\left(f\left(t, w_{n}(t)\right)-N(a(t), b(t), t)\right), \\
n & =0,1,2, \cdots
\end{aligned}
$$

2) The random problem (5) can be written as

$$
\begin{aligned}
z(t) & =g(t, x(t))-m(t, u(t)) \\
& -\eta(t)(f(t, w(t))-N(a(t), b(t), t)) \\
& +\left(I-\eta^{-1}(t)\right) R_{A(v(t), t)}(z(t)) .
\end{aligned}
$$

This random fixed point formulation is used to suggest the following algorithm:

Algorithm 2. For given $t \in \Omega, x_{0}(t), z_{0}(t) \in H$, $v_{0}(t) \in T\left(t, x_{0}(t)\right), \quad a_{0}(t) \in V\left(t, x_{0}(t)\right)$,

$b_{0}(t) \in G\left(t, x_{0}(t)\right), \quad w_{0}(t) \in P\left(t, x_{0}(t)\right)$,

$u_{0}(t) \in E\left(t, x_{0}(t)\right)$, compute $\left\{x_{n}(t)\right\},\left\{z_{n}(t)\right\}$,

$\left\{v_{n}(t)\right\},\left\{a_{n}(t)\right\},\left\{b_{n}(t)\right\},\left\{w_{n}(t)\right\}$ and $\left\{u_{n}(t)\right\}$ by random iterative schemes,

$$
\begin{gathered}
g\left(t, x_{n}(t)\right)=m\left(t, u_{n}(t)\right)+J_{A\left(v_{n}(t), t\right)}\left(z_{n}(t)\right), \\
v_{n}(t) \in T\left(t, x_{n}(t)\right), \\
\left\|v_{n+1}(t)-v_{n}(t)\right\| \leq \mathcal{H}\left(T\left(t, x_{n+1}(t)\right), T\left(t, x_{n}(t)\right)\right), \\
a_{n}(t) \in V\left(t, x_{n}(t)\right), \\
\left\|a_{n+1}(t)-a_{n}(t)\right\| \leq \mathcal{H}\left(V\left(t, x_{n+1}(t)\right), V\left(t, x_{n}(t)\right)\right), \\
b_{n}(t) \in G\left(t, x_{n}(t)\right), \\
\left\|b_{n+1}(t)-b_{n}(t)\right\| \leq \mathcal{H}\left(G\left(t, x_{n+1}(t)\right), G\left(t, x_{n}(t)\right)\right), \\
w_{n}(t) \in P\left(t, x_{n}(t)\right), \\
\left\|w_{n+1}(t)-w_{n}(t)\right\| \leq \mathcal{H}\left(P\left(t, x_{n+1}(t)\right), P\left(t, x_{n}(t)\right)\right), \\
u_{n}(t) \in E\left(t, x_{n}(t)\right), \\
\left\|u_{n+1}(t)-u_{n}(t)\right\| \leq \mathcal{H}\left(E\left(t, x_{n+1}(t)\right), E\left(t, x_{n}(t)\right)\right), \\
z_{n+1}(t)=g\left(t, x_{n}(t)\right)-m\left(t, u_{n}(t)\right) \\
\quad-\eta(t)\left(f\left(t, w_{n}(t)\right)-N\left(a_{n}(t), b_{n}(t), t\right)\right) \\
\quad+\left(I-\eta^{-1}(t)\right) R_{A\left(v_{n}(t), t\right)}\left(z_{n}(t)\right), \\
n=0,1,2, \cdots .
\end{gathered}
$$

\section{Main Result}

First we recall the following well known concepts.

Definition 5. A random mapping $g: \Omega \times H \rightarrow H$ is said to be

1) random strongly monotone, if there exists a measurable function $\delta: \Omega \rightarrow(0, \infty)$ such that

$$
\begin{aligned}
& \langle g(t, x(t))-g(t, y(t)), x(t)-y(t)\rangle \\
& \geq \delta(t)\|x(t)-y(t)\|^{2}, \text { for all } t \in \Omega, x, y \in H ;
\end{aligned}
$$

2) random Lipschitz continuous, if there exists a measurable function $\gamma: \Omega \rightarrow(0, \infty)$ such that

$$
\|g(t, x(t))-g(t, y(t))\|^{2} \leq \gamma(t)\|x(t)-y(t)\|^{2},
$$

for all $t \in \Omega, x, y \in H$.

Definition 6. A random mapping $V: \Omega \times H \rightarrow 2^{H}$ is said to be random relaxed monotone with respect to the first argument of a random mapping

$N: H \times H \times \Omega \rightarrow H$, if there exists a measurable function $\kappa: \Omega \rightarrow(0, \infty)$ such that

$$
\begin{aligned}
& \left\langle N\left(a_{1}(t), b(t), t\right)-N\left(a_{2}(t), b(t), t\right), x_{1}(t)-x_{2}(t)\right\rangle \\
& \geq-\kappa(t)\left\|x_{1}(t)-x_{2}(t)\right\|^{2},
\end{aligned}
$$

for all $t \in \Omega, \quad a_{i}(t) \in V\left(t, x_{i}(t)\right), \quad b(t) \in V(t, x(t))$, $i=1,2$.

Definition 7. A random mapping $G: \Omega \times H \rightarrow 2^{H}$ is said to be random relaxed Lipschitz continuous with respect to the second argument of random mapping

$N: H \times H \times \Omega \rightarrow H$ if there exists a measurable mapping $\alpha: \Omega \rightarrow(0, \infty)$ such that

$$
\begin{aligned}
& \left\langle N\left(a(t), b_{1}(t), t\right)-N\left(a(t), b_{2}(t), t\right), x_{1}(t)-x_{2}(t)\right\rangle \\
& \leq-\alpha(t)\left\|x_{1}(t)-x_{2}(t)\right\|^{2},
\end{aligned}
$$

for all $t \in \Omega, \quad a(t) \in G(t, x(t)), \quad b_{i}(t) \in G\left(t, x_{i}(t)\right)$, $i=1,2$.

Definition 8. A random set-valued mapping $E: \Omega \times H \rightarrow 2^{H}$ is said to be $\mathcal{H}$-Lipschitz continuous if there exists a measurable function $\sigma: \Omega \rightarrow(0, \infty)$ such that

$$
\begin{aligned}
& \mathcal{H}(E(t, x(t)), E(t, y(t))) \leq \sigma(t)\|x(t)-y(t)\|, \\
& \text { for all } t \in \Omega, x(t), y(t) \in H,
\end{aligned}
$$

where $\mathcal{H}$ is a Hausdorff metric on $H$.

Theorem 2. Let the random bifunction

$N: H \times H \times \Omega \rightarrow H$ be random Lipschitz continuous with respect to first and second arguments with measurable functions $r, s: \Omega \rightarrow(0, \infty)$ respectively and random set valued mappings $T, V, G, P, E: \Omega \times H \rightarrow 2^{H}$ be the random $\mathcal{H}$-Lipschitz continuous with random coefficients $v, \lambda, \xi, \rho, \sigma: \Omega \rightarrow(0, \infty)$ respectively. Let the random mappings $g, f, m: \Omega \times H \rightarrow H$ be random Lipschitz continuous with random coefficients

$\gamma, \mu, \beta: \Omega \rightarrow(0, \infty)$ respectively and the random mapping $g$ is the random strongly monotone with respect to the measurable map $\delta: \Omega \rightarrow(0, \infty)$. A random bifunction $N: H \times H \times \Omega \rightarrow H$ is randomly relaxed monotone with respect to the first argument of $N$ with random coefficient $k: \Omega \rightarrow(0, \infty)$ and relaxed Lipschitz continuous with respect to the second argument of $N$ with random coefficient $\alpha: \Omega \rightarrow(0, \infty)$. Let $A: H \times H \times \Omega \rightarrow 2^{H}$ be such that for each fixed $t \in \Omega$, 
$v(t) \in H, \quad A(., v(t), t): H \times \Omega \rightarrow 2^{H} \quad$ be a random maximal monotone satisfying

$g(t, x(t))-m(t, y(t)) \in \operatorname{DomA}(., v(t), t)$ for all $z(t), x(t), y(t) \in H$. Suppose that for any fix $t \in \Omega$, $x(t), y(t) \in H$,

$$
\left\|J_{A(x(t), t)} z(t)-J_{A(y(t), t)} z(t)\right\| \leq \epsilon(t)\|x(t)-y(t)\|, \quad(10)
$$

$$
\begin{aligned}
& \left|\eta(t)-\frac{(\kappa(t)+\alpha(t))-\mu(t) \rho(t)(1-p(t))}{(r(t) \lambda(t)+s(t) \xi(t))^{2}-(\mu(t) \rho(t))^{2}}\right| \\
& <\frac{\sqrt{(\kappa(t)+\alpha(t)-\mu(t) \rho(t)(1-p(t)))^{2}-(r(t) \lambda(t)+s(t) \xi(t))^{2}-(\mu(t) \rho(t))^{2} p(t)(2-p(t))}}{(r(t) \lambda(t)+s(t) \xi(t))^{2}-(\mu(t) \rho(t))^{2}} \\
& \quad \kappa(t)+\alpha(t)>\mu(t) \rho(t)(1-p(t))+\sqrt{(r(t) \lambda(t)+s(t) \xi(t))^{2}-(\mu(t) \rho(t))^{2} p(t)(2-p(t))}
\end{aligned}
$$$$
\kappa(t)+\alpha(t)>\mu(t) \rho(t)(1-p(t))
$$$$
\mu(t) \rho(t)<r(t) \lambda(t)+s(t) \xi(t), \quad p(t)<1
$$

where, $p(t)=q(t)+\varepsilon(t) v(t)$, $q(t)=2 \sqrt{1-2 \delta(t)+\gamma^{2}(t)}+2 \sigma(t) \beta(t), \quad q(t)<1$.

Then there exist $x(t), z(t) \in H, v(t) \in T(t, x(t))$,

$a(t) \in V(t, x(t)), \quad b(t) \in G(t, x(t)), \quad w(t) \in P(t, x(t))$, $u(t) \in E(t, x(t))$, satisfying (2) and (5) and random sequences $\left\{x_{n}(t)\right\},\left\{z_{n}(t)\right\},\left\{a_{n}(t)\right\},\left\{b_{n}(t)\right\},\left\{v_{n}(t)\right\}$, $\left\{w_{n}(t)\right\}$ and $\left\{u_{n}(t)\right\}$ generated by Algorithm 1, converge strongly to $x(t), z(t), v(t), a(t), b(t)$, $w(t)$ and $u(t)$ in $H$ for each $t \in \Omega$.

Proof. From Algorithm 1, we have

$$
\begin{aligned}
& \left\|J_{A(x(t), t)} Z(t)-J_{A(y(t), t)} Z^{\prime}(t)\right\| \\
& \leq\left\|z(t)-z^{\prime}(t)\right\|, \\
& \text { for all } z, z^{\prime} \in H, t \in \Omega,
\end{aligned}
$$

where $\varepsilon: \Omega \rightarrow(0, \infty)$ be the measurable map, then there exists $\eta: \Omega \rightarrow(0, \infty)$ such that

$$
\begin{aligned}
& \left\|z_{n+1}(t)-z_{n}(t)\right\|=\| g\left(t, x_{n}(t)\right)-g\left(t, x_{n-1}(t)\right)+m\left(t, u_{n}(t)\right)-m\left(t, u_{n-1}(t)\right) \\
& -\eta(t)\left(f\left(t, w_{n}(t)\right)-f\left(t, w_{n-1}(t)\right)-N\left(a_{n}(t), b_{n}(t), t\right)+N\left(a_{n-1}(t), b_{n-1}(t), t\right)\right) \| \\
& \leq\left\|x_{n}(t)-x_{n-1}(t)-\left(g\left(t, x_{n}(t)\right)-g\left(t, x_{n-1}(t)\right)\right)\right\|+\left\|m\left(t, u_{n}(t)\right)-m\left(t, u_{n-1}(t)\right)\right\| \\
& +\left\|x_{n}(t)-x_{n-1}(t)+\eta(t)\left(N\left(a_{n}(t), b_{n}(t), t\right)-N\left(a_{n-1}(t), b_{n-1}(t), t\right)\right)\right\| \\
& +\eta(t)\left\|f\left(t, w_{n}(t)\right)-f\left(t, w_{n-1}(t)\right)\right\| .
\end{aligned}
$$

Since $N$ is randomly Lipschitz continuous with respect to first and second argument and $V$ and $G$ are random $\mathcal{H}$ Lipschitz continuous, we have

$$
\begin{aligned}
& \left\|N\left(a_{n}(t), b_{n}(t), t\right)-N\left(a_{n-1}(t), b_{n-1}(t), t\right)\right\| \\
& \leq\left\|N\left(a_{n}(t), b_{n}(t), t\right)-N\left(a_{n-1}(t), b_{n}(t), t\right)\right\|+\left\|N\left(a_{n-1}(t), b_{n}(t), t\right)-N\left(a_{n-1}(t), b_{n-1}(t), t\right)\right\| \\
& \leq r(t)\left\|a_{n}(t)-a_{n-1}(t)\right\|+s(t)\left\|b_{n}(t)-b_{n-1}(t)\right\| \\
& \leq r(t) \mathcal{H}\left(V\left(t, x_{n}(t)\right), V\left(t, x_{n-1}(t)\right)\right)+s(t) \mathcal{H}\left(G\left(t, x_{n}(t)\right), G\left(t, x_{n-1}(t)\right)\right) \\
& \leq r(t) \lambda(t)\left\|x_{n}(t)-x_{n-1}(t)\right\|+s(t) \xi(t)\left\|x_{n}(t)-x_{n-1}(t)\right\| \\
& \leq(r(t) \lambda(t)+s(t) \xi(t))\left\|x_{n}(t)-x_{n-1}(t)\right\| .
\end{aligned}
$$


Again $m, f$ are randomly Lipschitz continuous and $T, P, E$ are random $\mathcal{H}$-Lipschitz continuous, we have

$$
\begin{aligned}
& \left\|m\left(t, u_{n}(t)\right)-m\left(t, u_{n-1}(t)\right)\right\| \leq \beta(t)\left\|u_{n}(t)-u_{n-1}(t)\right\| \\
& \leq \beta(t) \mathcal{H}\left(E\left(t, x_{n}(t)\right), E\left(t, x_{n-1}(t)\right)\right) \\
& \leq \sigma(t) \beta(t)\left\|x_{n}(t)-x_{n-1}(t)\right\| . \\
& \left\|f\left(t, w_{n}(t)\right)-f\left(t, w_{n-1}(t)\right)\right\| \leq \mu(t)\left\|w_{n}(t)-w_{n-1}(t)\right\| \\
& \leq \mu(t) \mathcal{H}\left(P\left(t, x_{n}(t)\right), P\left(t, x_{n-1}(t)\right)\right) \\
& \leq \mu(t) \rho(t)\left\|x_{n}(t)-x_{n-1}(t)\right\| . \\
& \quad\left\|v_{n}(t)-v_{n-1}(t)\right\| \leq \mathcal{H}\left(T\left(t, x_{n}(t)\right), T\left(t, x_{n-1}(t)\right)\right) \\
& \quad \leq v(t)\left\|x_{n}(t)-x_{n-1}(t)\right\| . \\
& \left\|g\left(t, x_{n}(t)\right)-g\left(t, x_{n-1}(t)\right)\right\| \leq \gamma(t)\left\|x_{n}(t)-x_{n-1}(t)\right\| .
\end{aligned}
$$

Since $g$ is random strongly monotone and from (18), we have

$$
\begin{aligned}
& \left\|x_{n}(t)-x_{n-1}(t)-\left(g\left(t, x_{n}(t)\right)-g\left(t, x_{n-1}(t)\right)\right)\right\|^{2} \\
& =\left\|x_{n}(t)-x_{n-1}(t)\right\|^{2} \\
& -2\left\langle g\left(t, x_{n}(t)\right)-g\left(t, x_{n-1}(t)\right), x_{n}(t)-x_{n-1}(t)\right\rangle \\
& +\left\|g\left(t, x_{n}(t)\right)-g\left(t, x_{n-1}(t)\right)\right\|^{2} \\
& \leq\left\|x_{n}(t)-x_{n-1}(t)\right\|^{2}-2 \delta(t)\left\|x_{n}(t)-x_{n-1}(t)\right\|^{2} \\
& +\gamma^{2}(t)\left\|x_{n}(t)-x_{n-1}(t)\right\|^{2} \\
& \leq\left(1-2 \delta(t)+\gamma^{2}(t)\right)\left\|x_{n}(t)-x_{n-1}(t)\right\|^{2} .
\end{aligned}
$$

Since $N$ is random relaxed monotone with respect to first argument and random relaxed Lipschitz continuous with second argument, we have

$$
\begin{aligned}
& \left\|x_{n}(t)-x_{n-1}(t)+\eta(t)\left(N\left(a_{n}(t), b_{n}(t), t\right)-N\left(a_{n-1}(t), b_{n-1}(t), t\right)\right)\right\|^{2} \\
& =\left\|x_{n}(t)-x_{n-1}(t)\right\|^{2}+2 \eta(t)\left\langle N\left(a_{n}(t), b_{n}(t), t\right)-N\left(a_{n-1}(t), b_{n-1}(t), t\right), x_{n}(t)-x_{n-1}(t)\right\rangle \\
& +\eta^{2}(t)\left\|N\left(a_{n}(t), b_{n}(t), t\right)-N\left(a_{n-1}(t), b_{n-1}(t), t\right)\right\|^{2} \\
& \leq\left\|x_{n}(t)-x_{n-1}(t)\right\|^{2}+2 \eta(t)\left\langle N\left(a_{n}(t), b_{n}(t), t\right)-N\left(a_{n-1}(t), b_{n}(t), t\right), x_{n}(t)-x_{n-1}(t)\right\rangle \\
& +2 \eta(t)\left\langle N\left(a_{n-1}(t), b_{n}(t), t\right)-N\left(a_{n-1}(t), b_{n-1}(t), t\right), x_{n}(t)-x_{n-1}(t)\right\rangle \\
& +\eta^{2}(t)\left\|N\left(a_{n}(t), b_{n}(t), t\right)-N\left(a_{n-1}(t), b_{n-1}(t), t\right)\right\|^{2} \\
& \leq\left\|x_{n}(t)-x_{n-1}(t)\right\|^{2}-2 \eta(t) \kappa(t)\left\|x_{n}(t)-x_{n-1}(t)\right\|^{2}-2 \eta(t) \alpha(t)\left\|x_{n}(t)-x_{n-1}(t)\right\|^{2} \\
& +\eta^{2}(t)(r(t) \lambda(t)+s(t) \xi(t))^{2}\left\|x_{n}(t)-x_{n-1}(t)\right\|^{2} \\
& \leq\left(1-2 \eta(t)(\kappa(t)+\alpha(t))+\eta^{2}(t)(r(t) \lambda(t)+s(t) \xi(t))^{2}\right)\left\|x_{n}(t)-x_{n-1}(t)\right\|^{2} .
\end{aligned}
$$

From (13)-(15), (19) and (20), we have

$$
\begin{aligned}
& \left\|z_{n+1}(t)-z_{n}(t)\right\| \\
& \leq\left(\sqrt{1-2 \delta(t)+\gamma^{2}(t)}+\sigma(t) \beta(t)+\sqrt{1-2 \eta(t)(\kappa(t)+\alpha(t))+\eta^{2}(t)(r(t) \lambda(t)+s(t) \xi(t))^{2}}+\eta(t) \mu(t) \rho(t)\right) \\
& \cdot\left\|x_{n}(t)-x_{n-1}(t)\right\| \\
& \leq\left[\frac{q(t)}{2}+\chi(\eta(t))+\eta(t) \mu(t) \rho(t)\right]\left\|x_{n}(t)-x_{n-1}(t)\right\|
\end{aligned}
$$

where $q(t)=2 \sqrt{1-2 \delta(t)+\gamma^{2}(t)}+2 \sigma(t) \beta(t)$ and

$$
\chi(\eta(t))=\sqrt{1-2 \eta(t)(\kappa(t)+\alpha(t))+\eta^{2}(t)(r(t) \lambda(t)+s(t) \xi(t))^{2}} .
$$


Also from (6), (10), (11), (15) and (19), we obtain

$$
\begin{aligned}
& \left\|x_{n}(t)-x_{n-1}(t)\right\|=\| x_{n}(t)-x_{n-1}(t)-g\left(t, x_{n}(t)\right)+g\left(t, x_{n-1}(t)\right)+m\left(t, u_{n}(t)\right) \\
& -m\left(t, u_{n-1}(t)\right)+J_{A\left(v_{n}(t), t\right)} z_{n}(t)-J_{A\left(v_{n-1}(t), t\right)}\left(z_{n-1}(t)\right) \| \\
& \leq\left\|x_{n}(t)-x_{n-1}(t)-\left(g\left(t, x_{n}(t)\right)-g\left(t, x_{n-1}(t)\right)\right)\right\| \\
& +\left\|m\left(t, u_{n}(t)\right)-m\left(t, u_{n-1}(t)\right)\right\|+\left\|J_{A\left(v_{n}(t), t\right)}\left(z_{n}(t)\right)-J_{A\left(v_{n-1}(t), t\right)}\left(z_{n}(t)\right)\right\| \\
& +\left\|J_{A\left(v_{n-1}(t), t\right)}\left(z_{n}(t)\right)-J_{A\left(v_{n-1}(t), t\right)}\left(z_{n-1}(t)\right)\right\| \\
& \leq \sqrt{\left(1-2 \delta(t)+\gamma^{2}(t)\right)}\left\|x_{n}(t)-x_{n-1}(t)\right\|+\sigma(t) \beta(t)\left\|x_{n}(t)-x_{n-1}(t)\right\| \\
& +\epsilon(t)\left\|v_{n}(t)-v_{n-1}(t)\right\|+\left\|z_{n}(t)-z_{n-1}(t)\right\| \\
& \leq\left(\sqrt{\left(1-2 \delta(t)+\gamma^{2}(t)\right)}+\sigma(t) \beta(t)+\epsilon(t) v(t)\right)\left\|x_{n}(t)-x_{n-1}(t)\right\|+\left\|z_{n}(t)-z_{n-1}(t)\right\| \\
& \leq\left(\frac{q(t)}{2}+\epsilon(t) v(t)\right)\left\|x_{n}(t)-x_{n-1}(t)\right\|+\left\|z_{n}(t)-z_{n-1}(t)\right\|
\end{aligned}
$$

which implies that

$$
\begin{aligned}
& \left\|x_{n}(t)-x_{n-1}(t)\right\| \\
& \leq \frac{1}{1-\frac{q(t)}{2}-\epsilon(t) v(t)}\left\|z_{n}(t)-z_{n-1}(t)\right\| .
\end{aligned}
$$

Adding (21) and (22), we obtain

$$
\begin{aligned}
& \left\|z_{n}(t)-z_{n-1}(t)\right\| \\
& \leq\left\{\frac{\frac{q(t)}{2}+\chi(\eta(t))+\eta(t) \mu(t)}{1-\frac{q(t)}{2}-\epsilon(t) v(t)}\right\}\left\|z_{n}(t)-z_{n-1}(t)\right\| \\
& \leq \theta(t)\left\|z_{n}(t)-z_{n-1}(t)\right\|,
\end{aligned}
$$

where,

$$
\theta(t)=\frac{\frac{q(t)}{2}+\chi(\eta(t))+\eta(t) \mu(t)}{1-\frac{q(t)}{2}-\epsilon(t) v(t)} .
$$

From (12), it follows that $\theta(t)<1$ for each $t \in \Omega$. Consequently from (23), we see that the random sequence $\left\{z_{n}(t)\right\}$ is a Cauchy sequence in $H$ for each $t \in \Omega$, that is there exists $z(t) \in H$ for fix $t \in \Omega$ with $z_{n+1}(t) \rightarrow z(t)$ as $n \rightarrow \infty$. From (22), we know that the random sequence $\left\{x_{n}(t)\right\}$ is a Cauchy sequence in $H$ that is there exists $x(t) \in H$ with $x_{n+1}(t) \rightarrow x(t)$ for each fix $t \in \Omega$. Also from the random Lipschitz continuity, we have

$$
\begin{aligned}
\left\|u_{n+1}(t)-u_{n}(t)\right\| & \leq \mathcal{H}\left(E\left(t, x_{n+1}(t)\right), E\left(t, x_{n}(t)\right)\right) \\
& \leq \sigma(t)\left\|x_{n+1}(t)-x_{n}(t)\right\| \\
\left\|v_{n+1}(t)-v_{n}(t)\right\| & \leq \mathcal{H}\left(T\left(t, x_{n+1}(t)\right), T\left(t, x_{n}(t)\right)\right) \\
& \leq v(t)\left\|x_{n+1}(t)-x_{n}(t)\right\| \\
\left\|w_{n+1}(t)-w_{n}(t)\right\| & \leq \mathcal{H}\left(P\left(t, x_{n+1}(t)\right), P\left(t, x_{n}(t)\right)\right) \\
& \leq \rho(t)\left\|x_{n+1}(t)-x_{n}(t)\right\| \\
\left\|a_{n+1}(t)-a_{n}(t)\right\| & \leq \mathcal{H}\left(V\left(t, x_{n+1}(t)\right), V\left(t, x_{n}(t)\right)\right) \\
& \leq \lambda(t)\left\|x_{n+1}(t)-x_{n}(t)\right\| \\
\left\|b_{n+1}(t)-b_{n}(t)\right\| & \leq \mathcal{H}\left(G\left(t, x_{n+1}(t)\right), G\left(t, x_{n}(t)\right)\right) \\
& \leq \xi(t)\left\|x_{n+1}(t)-x_{n}(t)\right\|,
\end{aligned}
$$

which implies that the random sequences $\left\{u_{n+1}(t)\right\}$, $\left\{v_{n+1}(t)\right\},\left\{w_{n+1}(t)\right\},\left\{a_{n+1}(t)\right\}$ and $\left\{b_{n+1}(t)\right\}$ are Cauchy sequences in $H$. Assume that $a_{n+1}(t) \rightarrow a(t) \in H, \quad b_{n+1}(t) \rightarrow b(t) \in H$, $v_{n+1}(t) \rightarrow v(t) \in H, \quad w_{n+1}(t) \rightarrow w(t) \in H \quad$ and $u_{n+1}(t) \rightarrow u(t) \in H$ as $n \rightarrow \infty$, for each fix $t \in \Omega$.

Now by using the random continuity of the random operators $g, m, f, T, V, G, P, E, N, J_{A}$ and Algorithm 1, we have

$$
\begin{aligned}
& z(t)=g(t, x(t))-m(t, u(t)) \\
& -\eta(t)(f(t, w(t))-N(a(t), b(t), t)) \in H .
\end{aligned}
$$

Now we show that $a(t) \in V(t, x(t))$. In fact 


$$
\begin{aligned}
& d(a(t), V(t, x(t))) \\
& \leq\left\|a(t)-a_{n}(t)\right\|+d\left(a_{n}(t), V(t, x(t))\right) \\
& \leq\left\|a(t)-a_{n}(t)\right\|+\mathcal{H}\left(V\left(t, x_{n}(t)\right), V(t, x(t))\right) \\
& \leq\left\|a(t)-a_{n}(t)\right\|+\lambda(t)\left\|x_{n}(t)-x(t)\right\| \rightarrow 0 \\
& \text { as } n \rightarrow \infty, \text { for fix } t \in \Omega
\end{aligned}
$$

where,

$$
d(a(t), V(t, x(t)))=\inf \{\|a(t)-z(t)\|: z \in V(t, x(t))\} .
$$

Since the random sequences $\left\{x_{n}(t)\right\}$ and $\left\{a_{n}(t)\right\}$ are Cauchy sequences, it follows from the above inequality that $d(a(t), V(t, x(t)))=0$. This implies that $a(t) \in V(t, x(t))$. In a similar way, we can show that $v(t) \in T(t, x(t)), \quad b(t) \in G(t, x(t)), \quad w(t) \in P(t, x(t))$ and $u(t) \in E(t, x(t))$.

By Theorem 1, it follows that $t \in \Omega, x(t), z(t) \in H$, $v(t) \in T(t, x(t)), a(t) \in V(t, x(t)), \quad b(t) \in G(t, x(t))$, $w(t) \in P(t, x(t))$ and $u(t) \in E(t, x(t))$, which satisfies the inequality (1) and for fix $t \in \Omega, z_{n}(t) \rightarrow z(t)$, $x_{n}(t) \rightarrow x(t), \quad v_{n}(t) \rightarrow v(t), \quad a_{n}(t) \rightarrow a(t)$, $b_{n}(t) \rightarrow b(t), \quad w_{n}(t) \rightarrow w(t)$ and $u_{n}(t) \rightarrow u(t)$ strongly in $H$, the required result.

\section{REFERENCES}

[1] C. Baiocchi and A. Capelo, "Variational and Quasi-Variational Inequalities,” John Wiley and Sons, New York, 1984.

[2] H. Brezis, “Operateurs Maximmaux Monotones,” North Holland, Amsterdam, 1973.

[3] F. Giannessi and A. Maugeri, "Variational Inequalities and Network Equilibrium Problems,” Plenum Press, New York, 1995.

[4] C. P. Toskos and V. J. Padgett, "Random Integral Equations with Applications to Stochastic Systems," SpringerVerlag, Berlin and New York, 1971.

[5] C. P. Toskos and V. J. Padgett, "Random Integral Equations with Applications in Life Sciences and Engineering,” Academic Press, New York, 1974.

[6] M. A. Noor, "Some Recent Advances in Variational Inequalities II,” New Zealand Journal of Mathematics, Vol. 26, No. 2, 1997, pp. 229-255.

[7] M. A. Noor, "Generalized Set-Valued Variational Inclu- sions and Resolvent Equations,” Journal of Mathematical Analysis and Application, Vol. 228, No. 1, 1998, pp. 206220. doi:10.1006/jmaa.1998.6127

[8] S. H. Shim, S. M. Kang, N. J. Huang and Y. J. Cho, "Generalized Set-Valued Strongly Nonlinear Quasi-Variational Inclusions," Indian Journal of Pure Applied Mathematics, Vol. 31, No. 9, 2000, pp. 1113-1122.

[9] S. S. Chang and N. J. Huang, "Generalized Random Multivalued Quasi Complementarity Problems,” Indian Journal of Mathematics, Vol. 35, No. 3, 1993, pp. 305-320.

[10] N. J. Huang, X. Long and Y. J. Cho, "Random Generalized Nonlinear Variational Inclusions," Bulletin of Korean Mathematical Society, Vol. 34, No. 4, 1997, pp. 603-615.

[11] T. Hussain, E. Tarafdar and X. Z. Yuan, "Some Results on Random Generalized Games and Random Quasi-Variational Inequalities,” Far East Journal Mathematical Society, Vol. 2, No. 1, 1994, pp. 35-55.

[12] N. X. Tan, "Random Quasi-Variational Inequalities,” Mathematische Nachrichten, Vol. 125, 1986, pp. 319-328.

[13] R. U. Verma, “On Generalized Variational Inequalities Involving Relaxed Lipschitz and Relaxed Monotone Operators," Journal of Mathematical Analysis and Application, Vol. 23, 1997, pp. 389-392.

[14] X. Z. Yuan, “Non Compact Random Generalized Games and Random Quasi-Variational Inequalities," Journal of Applied Stochastic Analysis, Vol. 7, No. 4, 1994, pp. 467486. doi:10.1155/S1048953394000377

[15] X. P. Ding and Y. J. Park, “A New Class of Generalized Nonlinear Implicit Quasi-variational Inclusions with Fuzzy Mappings,” Journal of Computer and Applied Mathematics, Vol. 138, No. 2, 2002, pp. 243-257. doi:10.1016/S0377-0427(01)00379-X

[16] A. Hassouni and A. Moudafi, “A Perturbed Algorithm for Variational Inclusions,” Journal of Mathematical Analysis and Application, Vol. 185, No. 3, 1994, pp. 706-712. doi:10.1006/jmaa.1994.1277

[17] N. J. Huang, “Generalized Nonlinear Variational Inclusions with Noncompact Valued Mappings,” Applied Mathematics Letter, Vol. 9, No. 3, 1996, pp. 25-29. doi:10.1016/0893-9659(96)00026-2

[18] L. U. Uko, "Strongly Nonlinear Generalized Equations," Journal of Mathematical Analysis and Application, Vol. 220, No. 2, 1998, pp. 65-76. doi:10.1006/jmaa.1997.5796

[19] Salahuddin, "Some Aspects of Variational Inequalities," Ph.D. Thesis, Aligarh Muslim University, Aligarh, 2000.

[20] Salahuddin and M. K. Ahmad, "On Generalized Multivalued Random Variational Like Inclusions,” Applied Mathematics, Vol. 2, No. 8, 2011, pp. 1011-1018. doi:10.4236/am.2011.28140 